\title{
Ciclo de supresión y retorno: Geografía simbólica de las literaturas indígenas
}

\author{
Supression and Return Cycle: Symbolic Geography of Indigenous Literatures
}

Ciclo de supressão e retorno: Geografia simbólica das literaturas indígenas

\section{Christopher B. Teuton}

UNIVERSITY OF WASHINGTON, ESTADOS UNIDOS

Profesor y Jefe del Programa American Indian Studies de Washington

University. PhD en Inglés con Minor en American Indian Studies,

University of Wisconsin-Madison. Es autor de Cherokee Stories of

the Turtle Island Liars' Club (University of North Carolina Press,

2012), Deep Waters: the Textual Continuum in American Indian

Literature (University of Nebraska Press, 2010), y coautor del

premiado Reasoning Together: the Native Critics Collective (University

of Oklahoma Press, 2008). Correo electrónico: teuton@unc.edu

\section{Artículo de reflexión}

Documento accesible en línea desde la siguiente dirección: http://revistas.javeriana.edu.co

\section{Traducción de Félix Ceballos}

(Profesional en filología e idiomas - Inglés, Universidad Nacional de Colombia, Postulante a Maestro en Literatura, Pontificia Universidad Javeriana. Profesor de inglés en el Proyecto de Internacionalización - Explora un Mundo (2013-2014), Unidad de Extensión de lenguas extranjeras, Departamento de Lenguas Extranjeras, Facultad de Ciencias Humanas (2013-presente), Universidad Nacional de Colombia. Ha realizado numerosas traducciones en literatura, estudios lingüísticos y análisis del discurso. Correo electrónico: flixceballos@gmail.com 


\section{Resumen}

Este ensayo ofrece un modelo para la comprensión de los patrones evolutivos de la narrativa que enmarca los movimientos literales y figurativos de personajes y argumentos en las literaturas indígenas. Concretamente, el análisis se apoya en un acercamiento que se deriva de los estudios del crítico literario afroamericano B. Stepto, esto con el fin de identificar un patrón cíclico de movimiento físico y psíquico en las literaturas indígenas que se manifiesta en lo que llamo narrativas de la supresión y narrativas del retorno. La siguiente geografía simbólica de las narrativas indígenas sitúa la experiencia indígena entre dos polos, a saber, el Centro simbólico y la Ciudad simbólica, los cuales se encuentran mediados por un tercer espacio, la Reserva simbólica.

Palabras clave: Literatura indígena; centro; ciudad; reserva simbólica

\section{Abstract}

This essay offers a model for the understanding of the evolutionary patterns of the narrative that frames the literal and figurative movements of characters and plot in indigenous literatures. Specifically, the analysis is based in an approach derived from the studies of the Afro-American literary critic B. Stepto, with the purpose of identifying a cyclical pattern of physical and psychic movement in the indigenous literatures, shown in what I call suppression narratives and return narratives. The following symbolic geography of indigenous narratives places the indigenous experiences between two poles, namely, the symbolic Center and the symbolic City, which are mediated by a third space, the symbolic Reserve.

Keywords: indigenous literature; center; city; symbolic reserve

\section{Resumo}

Este ensaio fornece modelo para a compreensão dos padrões evolutivos da narrativa que emoldura os movimentos literais e figurativos de personagens e argumentos nas literaturas indígenas. Concretamente, a análise apoia-se numa aproximação derivada dos estudos do crítico literário afroamericano B. Stepto, isso com o objetivo de identificar um padrão cíclico de movimento físico e psíquico nas literaturas indígenas manifestado no que chamo de narrativas da supressão e narrativas do retorno. A seguinte geografia simbólica das narrativas indígenas coloca a experiência indígena entre dois polos, que são o Centro simbólico e a Cidade simbólica, que estão mediados por um terceiro espaço, a Reserva simbólica.

Palavras-chave: Literatura indígena; centro; cidade; reserva simbólica

\section{Cómo citar este artículo:}

Teuton, Christopher. "Ciclo de supresión y retorno: Geografía simbólica

de las literaturas indígenas". Cuadernos de Literatura 19.38 (2015):

248-268. http://dx.doi.org/10.11144/Javeriana.cl19-38.csrg 
LA CRÍTICA LITERARIA indígena reciente se ha centrado amplia y acertadamente en modelos críticos derivados de la experiencia indígena oral y escrita. En la medida en que los estudios críticos de lo indígena continúen ampliando su alcance, se verán beneficiados por un diálogo con otras discusiones multiculturales y críticas a la vez que se mantendrán centradas en lo indígena. Este ensayo ofrece un modelo de comprensión de los patrones evolutivos que enmarcan los movimientos literales y figurativos de personajes y argumentos en las literaturas indígenas.

Concretamente, el análisis parte de un acercamiento que se deriva de los estudios del crítico literario afroamericano B. Stepto con el fin de identificar un patrón cíclico de movimiento físico y psíquico en las literaturas indígenas, que se manifiesta en lo que aquí será definido como narrativas de la supresión y narrativas del retorno. La consecuente geografía simbólica de las narrativas indígenas sitúa la experiencia indígena entre dos polos, a saber, el Centro simbólico y la Ciudad simbólica, los cuales se encuentran mediados por un tercer espacio, la Reserva simbólica. La búsqueda de una voz individual y de una comunitaria que está en conversación con ambas tradiciones, la indígena y la de la modernidad occidental, se desarrolla a través de una negociación de esta geografía simbólica que ilumina aspectos fundamentales de la experiencia indígena contemporánea.

Mi acercamiento a la geografía simbólica responde al paradigma expuesto por Stepto en su trabajo de 1970 que se titula: Behind the Veil: A Study of AfroAmerican Narrative. En este texto se enfatiza la importancia de la historia negra en las prácticas de producción de la literatura afroamericana. Stepto hace que la dialéctica entre esclavitud y libertad desempeñe un rol central en su modelo de historia de la narrativa afroamericana. El paradigma de Stepto, desarrollado ampliamente por Craig Hansen Werner, Farrah Jasmine Griffin, Hazel Carby, Edward M. Pavlic y Paul Gilroy, se construye alrededor de la tensión entre lo que él identifica como el sur y el norte simbólicos (167). El sur simbólico es el origen de la historia y la cultura afroamericanas; como espacio psíquico o literal, puede existir en cualquier sitio en tanto una comunidad afroamericana se reúna bajo condiciones de opresión, de Mississippi a Harlem. En la misma medida en que es una fuente de solidaridad y sentido de pertenencia familiar y cultural, el sur es también el sitio de máxima esclavitud para aquellos afroamericanos que se hayan en una cultura blanca históricamente racista. La pobreza, el crimen y el drama de una sociedad disfuncional son aspectos que hacen parte de la vida diaria en el sur simbólico; allí donde el poder institucional es controlado por euroamericanos. El norte simbólico ofrece "la estructura social menos opresiva que el mundo de la narrativa podía permitirse" $\left({ }_{167}\right)$. Su seguridad relativa, su oportunidad económica 
y su movilidad social son atractivas, pero van en detrimento de la comunidad afroamericana.

El análisis de la cultura oral y de las narrativas esclavas propuesto por Stepto [...] genera dos patrones centrales: la narrativa del ascenso, en la que el protagonista progresa hacia la educación y la libertad (usualmente en el norte), y la narrativa de la inmersión, en la que el protagonista retorna a sus raíces culturales y se reintegra con su comunidad (usualmente en el sur). (Werner 29)

El héroe de la narrativa del ascenso, en su búsqueda, abandona el sur simbólico como objeto de la esclavitud (tanto metafórica como literal), una figura casi analfabeta para la cual el norte ofrece la promesa de una verdadera libertad. Empleando términos que más adelante ajustaré a los intereses de este estudio, Stepto bautiza al protagonista de la narrativa del ascenso con el nombre de "sobreviviente articulado". El sobreviviente articulado ha obtenido acceso a la libertad relativa del Norte simbólico a través de la adquisición del "alfabetismo", lo cual supone la habilidad de leer no solo palabras, sino, también, los signos y reglas del poder que hacen parte, con frecuencia implícitamente, de la sociedad del Norte simbólico. La narrativa del ascenso crea una existencia aislada o alienada en el norte, la cual motiva al sobreviviente articulado a volver al Sur simbólico en busca de su familia, su comunidad, su historia, su cultura y sus raíces. El sobreviviente articulado que retorna al Sur simbólico y se convierte en una autoridad en sus tradiciones ya que adquiere de nuevo una "alfabetización tribal", es identificado por Stepto como un "sujeto articulado al clan, la familia o la comunidad" (167).

$\mathrm{Al}$ adaptar el método de Stepto a las particularidades de la experiencia indígena, identifiqué tres ubicaciones geográficas simbólicas que desempeñan un rol central en numerosos textos indígenas: el Centro simbólico, la Ciudad simbólica y la Reserva simbólica. En lo que sigue definiré las características de cada una de estas zonas y rastrearé las relaciones entre las mismas, buscando identificar dos paradigmas análogos a las narrativas del ascenso y la inmersión de Stepto: las narrativas de supresión ${ }^{1}$ y las narrativas del retorno. En el presente texto, me referiré a un amplio rango de narrativas indígenas canónicas y contemporáneas. Propongo esta geografía simbólica no como un modelo reductivo, sino con el

1 La palabra empleada por el autor es removal que bien puede traducirse como remoción. He decidido emplear el término supresión porque considero que tiene una gama semántica mucho más amplia y que contiene el hecho violento del desplazamiento forzado, sea por la guerra o la presión cultural. Supresión es alienación, segregación y desplazamiento forzado, además de contener un matiz cultural muy fuerte cuando se piensa en la supresión de tradiciones e identidades. N. del T. 
interés de ofrecer un vocabulario que garantice lecturas más profundas en las complejidades de los textos indígenas, así como para presentar un marco de trabajo por medio del cual estudiar la manera en que las narrativas culturales se desarrollan en una relación "dialéctica" y de "contrapunto", lo que Stepto define como "llamadas" y "respuestas" entre textos en ambas narrativas, de forma y contenido. Mi modelo no es el primero en señalar la importancia de ubicaciones geográficas recurrentes en los textos indígenas; William Bevis, Robert Dale Parker, Michael D. Wilson, y Sean Kicummah Teuton proporcionaron cada uno sus teorías en relación con los lugares y movimientos de los personajes indígenas. La geografía simbólica que propongo conceptualiza la forma en que las narrativas indígenas se constituyen unas a otras, contribuyendo así al entendimiento, no solo de las literaturas indígenas, sino, también, de la experiencia indígena misma.

\section{El Centro simbólico, la Ciudad simbólica y la Reserva simbólica}

Así como el Sur simbólico y el Norte simbólico se encuentran mutuamente definidos por su relación con la historia de la esclavitud afroamericana, la Reserva y la Ciudad simbólicas existen en una relación dialéctica que surge del impacto del colonialismo occidental en Norte América. La discusión de la geografía simbólica en las literaturas indígenas debería empezar con un reconocimiento del Centro simbólico. El Centro simbólico de una nación indígena es un sitio literal a la vez que un espacio psíquico. En lo literal, se refiere a la zona -el paisaje o terreno mismo- que un pueblo usa para definir su sitio de origen. En términos psíquicos, es el espacio en el que un pueblo se origina y continúa autodefiniéndose a través de patrones específicos de pensamiento cultural. El centro es donde el mito se asocia al sitio; donde los patrones de significado cultural toman forma; donde la tradición como un eslabón indeleble entre pasado y presente se mantiene. En el Centro simbólico las leyes de la sociedad y de la creación son comprensibles, las tradiciones culturales dan cuenta del cambio y el crecimiento y las experiencias del día a día y los patrones de desarrollo cultural y personal son reconocibles positivamente y tienen significado. El Centro simbólico es una matriz de procesos culturales que describe de modo colectivo una tradición crítica tribal que puede dar cuenta del crecimiento, el cambio y la continuidad de una comunidad dentro de ambientes y lugares específicos.

En contraste con la mayoría de representaciones de la vida indígena tradicional en la cultura popular, las literaturas indígenas contemporáneas no retratan el Centro simbólico en una forma culturalmente "pura" o inalterada. Las culturas indígenas cambian con el tiempo y como el estudioso Choctaw Michael D. Wilson argumenta en su trabajo acerca del pensamiento de los indios Pueblo, que 
el centro nunca es estático, se trata de un "centro de valor estable a la vez que mutable" (Wilson, "Speaking" 136). Como un proceso dinámico de conciencia humana que puede referenciarse en la realidad física a través de los lugares, artefactos espirituales o relatos, el Centro simbólico en la literatura indígena puede representarse en incontables formas. En Black Elk Speaks, el "aro de la nación", como símbolo de la solidaridad política, cultural y espiritual de los Oglala Sioux, se constituye como Centro simbólico. En The Way to Rainy Mountain de N. Scott Momaday, el sitio real de la Montaña lluviosa así como las lecciones que se aprenden en el proceso de la migración de los Kiowase se establecen como el Centro simbólico de esta narrativa. En Ceremony de Leslie Marmon Silko, la kiva es el Centro simbólico, mientras que en The Grass Dancer de Susan Power, el Powwow mismo representa el Centro simbólico en movimiento. Para María en Halfbreed de María Campbell, se accede al Centro simbólico por medio de las conversaciones que tiene con su Cheechum; para Lisa en Monkey Beach de Eden Robinson, se puede acceder al Centro simbólico a través del espíritu embaucador de un puerco. Como el origen dominante del indigenismo de una cultura, la presencia o ausencia del Centro simbólico se traduce en una fuerza irreductible en la literatura indígena.

La segunda ubicación central en la geografía simbólica de la literatura indígena es la Ciudad simbólica, el espacio cosmopolita literal y/o figurativo en el cual los valores dominantes de la cultura colonial occidental se manifiestan y privilegian. Las imágenes de la Ciudad simbólica en la literatura indígena se estructuran alrededor de amplios patrones históricos; el interés se centra más en la presentación de un sentido claro de las fuerzas involucradas que en la profundización de las complicaciones y matices internos de la tradición occidental. Como producto de la modernidad, la Ciudad simbólica abarca una noción progresiva de la historia que se relaciona de forma discontinua con el pasado. Su religión es la cristiandad, la religión de las Escrituras. En un esfuerzo por acercarse a la tradición textual, se accede al conocimiento secular y su autoridad a través de palabras sobre el papel y se domina por medio del pensamiento científico. La Ciudad simbólica es el espacio del homo economicus que en esencia, obtiene su fuerza del consumo masivo, la reificación de los valores individuales y las conveniencias políticas colonialistas del capitalismo tardío. En su manifestación de espacio urbano real, existe como Los Ángeles de Abel en House Made of Dawn de Momaday; como el Vancouver de María en Halfbreed de Campbell; como la Minneapolis de Simón en The Hiawatha de David Treuer; como el Fort Simmer de Larry en The Lesser Blessed de Van Camp y como la Seattle de la ficción de Sherman Alexie. 
La Ciudad simbólica no se limita al espacio urbano de la realidad. Puede transportarse a través de los personajes e instituciones y manifestarse donde sea que los valores de la Ciudad simbólica echen raíces. En los mundos narrativos de las literaturas indígenas, la Ciudad simbólica se expresa donde y cuando los personajes se encuentren con el colonialismo de occidente y sus valores de modernidad. En Ceremony de Silko, la Ciudad simbólica se ve representada en la herida abierta que es el cañón de la mina de uranio en la concesión de la tierra de Cebolleta, así como los peligrosos valores occidentales que los veteranos de la Segunda Guerra Mundial de Laguna traen de vuelta al pueblo. George Jim en el relato de Harry Robinson "An Okanagan Indian Becomes a Captive Circus Show piece in England" nunca escapa de la Ciudad simbólica de Inglaterra. Este espacio destruye la inocencia y pervierte la fe de Jeremías y Gabriel en el colegio residencial de Birch Lake en Kiss of the Fur Queen de Tomson Highway. La influencia de la Ciudad simbólica conduce al jefe del Clan del Venado, Bud Tunny, a la locura en Smoke Dancing de Eric Gansworth, como lo hace con el jefe Choctaw Redford Mcalester en Shell Shaker de Le Anne Howe.

La tercera de las ubicaciones centrales, la Reserva simbólica, es un espacio de intersección indígena en el cual las relaciones históricas entre el empuje del colonialismo occidental y las aserciones de soberanía cultural indígena desempeñan un rol muy importante en la vida y las experiencias cotidianas de los miembros de la comunidad. La Reserva simbólica no se limita al sitio literal, la reserva definida por los sistemas políticos en los que los pueblos indígenas existen, por el contrario, se trata de un sitio donde los valores indígenas y occidentales se enfrentan en un contexto comunitario indígena. El término reserva sirve de marcador político para aquello contra lo que la Reserva simbólica se resiste: una historia de opresión política y cultural manifiesta en la pérdida de territorio tradicional y la limitación de movimiento, literal y figurativo y el ataque a los valores tribales. Las comunidades de la Reserva simbólica negocian una tensión entre vivir de acuerdo a los valores del Centro simbólico y lidiar con la Ciudad simbólica con el fin de sobrevivir. La Reserva simbólica no es así, un espacio cultural o político puro, sino uno en el cual los personajes perciben simultáneamente un apoyo al sentido de indigenidad por parte de la comunidad y un ataque de las presiones coloniales internalizadas.

En la Reserva simbólica las tensiones entre los valores indígenas tradicionales y la modernidad son a menudo paradójicas. A la vez que un sitio de empobrecimiento económico generalizado es también, un sitio de libertad relativa con respecto a los imperativos consumistas de la cultura occidental. A la vez que las tradiciones orales son valoradas en la Reserva simbólica, el espacio es 
objeto de la ley escrita de la colonización occidental. Los personajes en la Reserva simbólica valoran el parentesco, pero su movimiento es limitado por las responsabilidades familiares, de clan y de religión. Sus miembros siguen la fe tradicional de su comunidad o las prácticas espirituales cristianas o una mezcla sincrética de creencias. En la Reserva simbólica se afirma la soberanía cultural y nacional indígena, pero esta es constantemente desafiada por los agentes de los gobiernos coloniales. Estas paradojas son parte de la realidad que se vive en la Reserva simbólica, allí se privilegia la tradición como un vínculo continuo entre el pasado y el presente entendidos, experiencialmente, a través de tradiciones orales, parentesco y espiritualidad. Estos mismos valores son, no obstante, permanentemente amenazados y socavados por la lucha con el colonialismo y la modernidad dentro de los terrenos físico y psíquico.

La propia América del Norte es un sitio de Reserva simbólica en tanto que se integra por numerosas tierras indígenas que se diferencian solo por el grado de cuestionamiento que hay del título América del Norte desde los diferentes actores: el gobierno nacional colonial y el indígena. Ambos, el título indígena de los territorios y el modo de vida indígena en estos espacios, se encuentran bajo amenaza colonial en tierras que en la actualidad pertenecen a las comunidades indígenas. Es por esta razón que la Reserva simbólica existe de manera evidente en reservas y en tierras tribales en las literaturas indígenas. Sin embargo, así como esta noción se mueve en la conciencia de los personajes y se intuye dentro de los mundos narrativos de estas literaturas, también existe en nichos específicamente tribales al interior de las comunidades en áreas urbanas; en la intertribal e itinerante comunidad Powwow; en las pantribales e intertribales organizaciones religiosas de las comunidades; y en las comunidades de las organizaciones profesionales, por hacer algunas menciones. Como sitio de mediación política y cultural entre el Centro simbólico y la Ciudad simbólica, la Reserva simbólica provee el contexto geográfico simbólico más común para las narrativas indígenas. Gabriel y Jeremiah en Kiss of the Fur Queen de Highway luchan para hacerse una vida significativa a través del arte dentro de la Reserva simbólica de Winnipeg y Toronto. En The Heartsong of Charging Elk de James Welch, Charging Elk vive en la Reserva simbólica de Marseilles. En Dead Voices, Gerald Vizenor explora nuevas posibilidades de vida indígena en las experiencias de Bagese en la Reserva simbólica de Oakland, California. Del mismo modo, Winona LaDuke explora este tipo de experiencias en la Reserva de White Earth en Last Standing Woman.

Las interacciones entre el Centro simbólico, la Ciudad simbólica y la Reserva simbólica pueden imaginarse en relación con el modelo dialéctico de la doble-consciencia que fue reseñado por primera vez por el académico afroamericano W.E.B. 
Du Bois en su trabajo The Souls of Black Folk. Du Bois ubica a los euroamericanos y a los afroamericanos en una dialéctica de oposición separada apenas por un "velo". El velo se encuentra contenido por discursos dominados por las narrativas euroamericanas que definen y categorizan a los afroamericanos en términos racistas, sexistas y primitivistas. El reto está en identificar y entender las narrativas que apoyan estos discursos de injusticia para luego reemplazar el velo con narrativas alternativas que subviertan o como Du Bois lo entiende, los trasciendan. Esta propuesta se encuentra implícita en los fundamentos de la Geografía simbólica de Stepto, la cual se encuentra configurada alrededor de la oposición binaria entre Norte y Sur simbólicos. En la literatura indígena la situación se complica debido a la presencia de un tercer espacio. Uno podría imaginar la tensión entre el Centro simbólico y la Ciudad simbólica en términos de Du Bois, pero la presencia de la Reserva simbólica crea una disrupción en esta relación binaria. La dialéctica entre la Ciudad y el Centro simbólicos es mediada por la Reserva simbólica. Allí, las narrativas dominantes del colonialismo y la modernidad que se generan en la Ciudad simbólica, se contrarrestan, se subvierten y se refiguran por las perspectivas de la realidad del Centro simbólico.

La interacción entre las zonas geográficas simbólicas se estructura a partir de la dialéctica entre la Ciudad y el Centro simbólicos. En los mundos narrativos de las literaturas indígenas el Centro simbólico a menudo se asimila como un espacio descentrado por los efectos del colonialismo occidental y la modernidad sobre sus personajes y sus comunidades. El colonialismo destruyó en su totalidad gobiernos nacionales indígenas o desplazó las naciones tribales soberanas. La modernidad, con su historia teleológica y su fe en el capitalismo, creó un cisma entre el pasado y el presente indígenas, iniciando así un reemplazo de la tradición cultural por la historia progresiva, la imposición de los valores individualistas de occidente y la reificación de explicaciones científicas racionales por encima de las relaciones holísticas con la naturaleza. Se hace necesario el restablecimiento de un canal de comunicación entre el Centro simbólico y la Ciudad simbólica con el fin de dar cabida a discursos alternativos. La salud del Centro simbólico depende de la subversión de los discursos occidentales que interrumpieron la soberanía de los pueblos indígenas y que menospreciaron su comprensión de la realidad. Irónicamente, la supervivencia de la Ciudad simbólica depende de la recuperación de un sentido de humanidad que esté en la capacidad de comprender las interrelaciones que hay entre todas las diferentes clases de vida, un propósito en el cual una relación con el Centro simbólico puede resultar beneficiosa. De esta manera, el establecimiento de relaciones entre el Centro simbólico y la Ciudad simbólica es de crucial importancia para ambas zonas. 
Como espacio de convergencia, la Reserva simbólica media entre la Ciudad y el Centro simbólicos (Fig. 1). A la vez que absorbe fuerza cultural del Centro, su necesidad de negociar con la Ciudad simbólica a fin de asegurar la supervivencia de la propia Reserva causa con frecuencia tensiones con los valores del Centro. De forma similar, la Ciudad simbólica influye en la Reserva simbólica a través de su economía, política y cultura popular, de lo que la Reserva simbólica hace parte a la vez que reconfigura esta dinámica, afirmando su soberanía. Es al interior de la Reserva simbólica que se pueden concebir y emplear las nuevas narrativas alternativas al discurso de la dominación colonial y la modernidad occidental. Con el fin de que esas narrativas se desarrollen, los individuos deben comunicarse con fluidez en las lenguas de ambos espacios simbólicos, del Centro y de la Ciudad, de manera que se cree un movimiento de vaivén entre las zonas culturales y sus vocabularios. Como aquí se demostrará luego, las narrativas de la supresión y del retorno representan las vías en las que el conocimiento del Centro simbólico y el de la Ciudad simbólica inician un diálogo a través de la experiencia de los individuos que se están moviendo entre estas dos zonas.

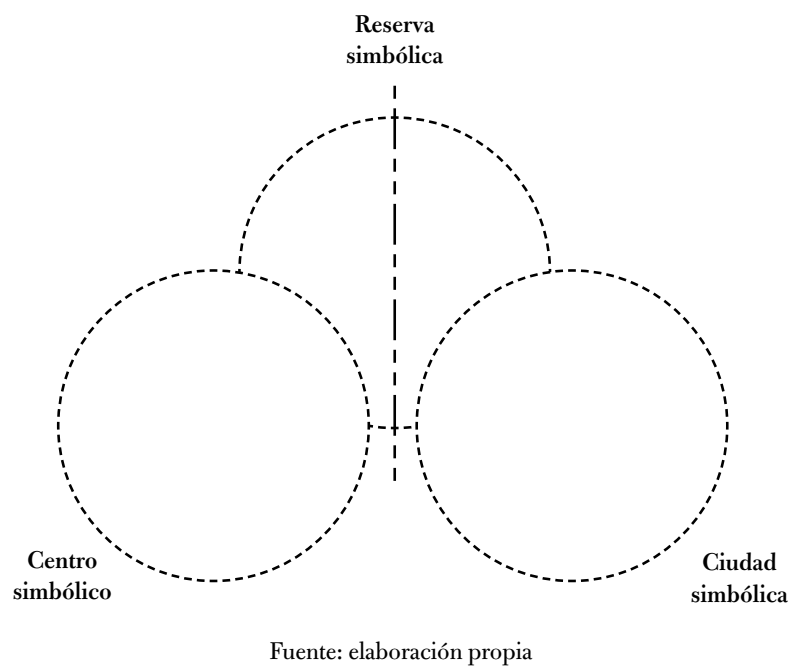




\section{Narrativas de la supresión y del retorno}

La geografía simbólica de las literaturas indígenas configura espacios a través de los movimientos literales y psíquicos de los personajes entre y dentro del Centro, la Reserva y la Ciudad simbólicas en tanto que triunfan o fracasan en adquirir una "voz", o un punto de vista cultural desde el cual poder nombrar la génesis de un cosmos. Con frecuencia, esta voz representa un intento de legitimación de los valores del Centro simbólico en un mundo que se encuentra estructurado por los valores de la Ciudad simbólica. Pero las dinámicas de la voz son contradictorias, al callar se busca un repudio a la modernidad, a la vez, la voz activa desentraña los aspectos potencialmente útiles de la Ciudad simbólica y los aprovecha al servicio de las necesidades del individuo y de su comunidad. Mientras que el velo de Du Bois inhibe la vista, el velo en las literaturas indígenas inhibe el sonido, impidiendo a los personajes hablarse y escucharse en su propia lengua, tanto de forma literal como metafórica. Las narrativas de la supresión y del retorno emplean el concepto de articulación figurado por el tropo de la voz como medio para representar la fluidez de un individuo en la lengua dominante de una zona geográfica simbólica específica. La adquisición de voz por parte de un individuo le asegura su supervivencia y su independencia en términos comunicativos de palabras, señas y reglas de poder local. Este no es un asunto sencillo, puesto que, como explica Louis Owens con respecto a las literaturas indígenas,

[...] el conflicto se exacerba a través de discursos antagónicos, a través del rompimiento entre la comunicación y el entendimiento, es decir, en el fracaso de la articulación de discursos. En una confrontación con la voz autoritaria, la privilegiada de la América Europea, el indígena recurre a la subversión o, con frecuencia, calla. (8)

El protagonista de ambas narrativas, la de supresión y la de retorno, debe negociar el velo de discurso al cual Owens alude. Con base en lo dicho por Stepto, los personajes que buscan articularse con el lenguaje de la Ciudad simbólica se denominan supervivientes articulados, mientras aquellos que pierden su voz son supervivientes desarticulados.

Los individuos que obtienen fluidez en los discursos de la Reserva simbólica se convierten en "individuos articulados con el clan o comunidad", mientras que aquellos quienes fracasan en su negociación lingüística se convierten en "individuos desarticulados de su clan o comunidad". El estudio de los patrones de los ciclos narrativos de supresión y retorno nos permite entender mejor las elecciones que los individuos hacen en tanto se mueven entre las tensiones creadas por la Ciudad, la Reserva y el Centro simbólicos. 
La historia colonial norteamericana de la supresión o desplazamiento de los pueblos indígenas de sus tierras a través de guerras de limpieza étnica, adoctrinamiento forzoso en los colegios y políticas legislativas diseñadas para borrar la identidad tribal proporciona el contexto para las narrativas de la supresión. Con cada relato de abandono de la Reserva simbólica, los personajes indígenas repiten de manera individual y simbólica aquello a lo que las comunidades de los pueblos estuvieron sometidas desde la colonización. El protagonista de la narrativa de la supresión abandona la Reserva simbólica como resultado de la presión de fuerzas externas o por decisión personal (lo cual no se encuentra enteramente desligado de la presión de dichas fuerzas).

Los individuos abandonan la Reserva por razones económicas, tales como la escasez de trabajo; la necesidad de escapar de una vida de luchas en la reserva; el deseo de obtener una educación occidental; y la compulsión psicológica y cultural de vivir lejos del hogar iniciada en la época de la alfabetización forzosa en los colegios. Dentro de la lógica de la misión ${ }^{2}$, el protagonista de las narrativas de la supresión viaja a la Ciudad simbólica en busca de la obtención de conocimiento que resulta importante para el desarrollo de su sentido de individuo y para el desarrollo del sentido de pertenencia de su comunidad en la Reserva simbólica. La narrativa de la supresión no deja de representar un movimiento ambiguo positivo. El precio del abandono de la Reserva simbólica es la pérdida de la solidaridad cultural y política con la propia comunidad.

La narrativa del retorno deriva su estructura de dos vertientes, las búsquedas míticas tradicionales indígenas y la realidad histórica de las separaciones y reuniones que los individuos indígenas y sus comunidades han experimentado como consecuencia y condición del colonialismo. El protagonista del viaje de retorno abandona la Ciudad simbólica (en ocasiones de manera voluntaria, otras veces por necesidad) en busca de un escape de una existencia que es, para invocar el lenguaje que Stepto emplea para describir al superviviente articulado en el Norte simbólico, "en el mejor de los casos, una existencia de soledad; en el peor de los casos, una de alienación" (167). Su misión es la de traer de vuelta

2 La palabra empleada por el autor es quest, una palabra que en inglés y específicamente en las literaturas mitológicas y legendarias, se entiende como una misión, pero que es a la vez una búsqueda. He decidido emplear la palabra misión por el aspecto de aventura que supone el salir de la comunidad indígena para integrarse a la ciudad moderna; bien puede tratarse de una misión de ida y vuelta en busca de un conocimiento, una negociación o un bien. La acepción búsqueda he querido dejarla de lado puesto que, a pesar de tratarse de una búsqueda, la palabra no contiene los matices que integren la idea de misión, aspecto que la última sí contiene con respecto a la búsqueda. $\mathrm{N}$ del $\mathrm{T}$. 
conocimiento de la Ciudad simbólica y reunirse, literalmente o simbólicamente, con la comunidad de la Reserva simbólica, que ha estado perdiendo miembros frente a la Ciudad. El viaje se inicia principalmente, para escapar a problemas legales. Este desplazamiento puede tomar muchas formas: física, espiritual, psicológica o política; con frecuencia, tiene todas las formas antes citadas. En tanto la narrativa del retorno es una búsqueda en la que la fe es un elemento inherente, sus resultados son inciertos. El éxito o el fracaso de la misión depende de la habilidad del personaje para aprender el lenguaje de la Ciudad simbólica y equilibrar efectivamente las influencias que el conocimiento aprendido así ejerce sobre su persona con las experiencias nuevas que obtiene en el Centro simbólico. El héroe exitoso de la narrativa del retorno sacrifica su libertad relativa como dimensión cultural que aísla la subjetividad de un miembro de una tribu y comunidad con cultura, tradición, historia y compromisos políticos.

\section{De superviviente a persona de clan: De desarticulado a articulado}

Las narrativas indígenas pueden empezar en cualquier punto del ciclo de la supresión y del retorno. Algunas narrativas empiezan con un personaje que ha aprendido, por lo menos de manera provisional, a equilibrar las visiones de mundo en oposición desde el Centro simbólico y la Ciudad simbólica. Otras narrativas describen a un personaje que aún no ha logrado este equilibrio. Como consecuencia, las narrativas de la supresión y del retorno siguen diferentes trayectorias. La narrativa de la supresión describe a una persona desarticulada de su clan que se desplaza hacia la Ciudad simbólica, donde puede aprender y comunicarse en el lenguaje de esa zona y convertirse en un superviviente articulado. Si esta misión no se completa, un superviviente articulado o desarticulado retorna a la Reserva simbólica para iniciar el proceso de aprendizaje de comunicación en el lenguaje de la Reserva y para compartir aquello que tenga para ofrecer, lo cual es usualmente, su conocimiento acerca de la Ciudad simbólica. El éxito o fracaso de la misión depende de la adquisición de conocimiento por parte del personaje, un conocimiento que le permita entender la importancia del Centro simbólico y del rol que él mismo desempeñará en este proceso.

El individuo desarticulado de su clan o tribu se encuentra inhabilitado para mediar en las negociaciones entre las influencias de la Ciudad y el Centro simbólicos en la Reserva simbólica y por cuenta de este desequilibrio, la confusión, la incertidumbre y la volatilidad amenazan la unidad de la comunidad. El desafío para los personajes que habitan esta zona es el de aprender cómo y por qué las paradojas de la Reserva simbólica existen; llegar a un entendimiento de que estas paradojas son condiciones derivadas del colonialismo y no marcas de debilidad que se reflejan como aspectos de inferioridad inherentes a las visiones de mundo de 
las comunidades indígenas. La dimensión cognitiva de este individuo manifiesta disonancias a través de malinterpretaciones de los sentidos del Centro simbólico, así como de problemas con el sistema legal, el cual se encuentra asociado a la Ciudad simbólica. El individuo Desarticulado de su clan es potencialmente peligroso para la comunidad de la Reserva simbólica puesto que se encuentra desarraigado a pesar de que habita en la zona de la Reserva. Personajes tales como Abel en House Made of Dawn, Tayo en Ceremony y Bud Tunny en Smoke Dancing malentienden las tradiciones del Centro simbólico de sus comunidades y reaccionan violenta e ilegalmente.

El individuo desarticulado de su clan está claramente delimitado en el texto por una falta de voz que lo paraliza, lo cual resulta en una metáfora que apunta a una falta de perspectiva cultural desde la cual generar un mundo posible. D'Arcy McNickle escribe en la introducción a The Surrounded:

Se sentaron un rato en silencio. Era inútil hablar acerca de malabarismos, pero por un largo instante Archilde no pudo pensar en nada que no fuera igualmente inútil. Cuando regresabas a casa, a tu madre indígena, era menester recordar que aquello era un mundo muy diferente. De cualquier modo, no habías regresado para presumir con tu dinero y hablar de ti mismo. (3)

En Ceremony, Tayo habla de sí mismo al doctor del ejército que lo está atendiendo: "[...] no puede hablarte. Es invisible. Sus palabras están formadas por una lengua invisible, no tienen sonido alguno" (Silko 14). Momaday escribe acerca de Abel en House Made of Dawn:

Ojalá hubiera podido decirlo, cualquier cosa en su propio idioma -incluso esa conocida formula de saludo $<_{\dot{d}} A$ dónde vas? $>$ - cualquier cosa que no hubiese traspasado el sonido, que no tuviera sustancia visible alguna, aquello hubiera podido revelarlo entero de nuevo a sí mismo. Pero estaba atontado. No atontado -el silencio seguía siendo la mejor parte de las antiguas costumbres- sino desarticulado. (53)

Como Rocky en Ceremony que asegura que el conocimiento tradicional de los Laguna no es más que una superstición, el individuo desarticulado de su clan que se ha desplazado a la Ciudad simbólica empieza a creer en los juicios de valor occidentales con respecto al pensamiento indígena. En Black Elk Speaks, Black Elk motiva su desplazamiento a la Ciudad simbólica:

Me volví al pasado y recordé las antiguas costumbres de mi pueblo, pero mi pueblo ya no vivía de aquella manera. Ahora caminaban por el camino negro, en soledad y sin objeto alguno, como en mi visión. El desespero me llevó a 
pensar que si los Wasichus parecían tener una mejor vida, tal vez mi pueblo debería seguir esas costumbres. Ahora sé que pensar así fue una tontería, pero era joven y estaba desesperado. (Neihardt 215)

Como héroe de la narrativa de la supresión, el individuo desarticulado de su clan busca autoconocimiento a través de un mayor entendimiento de las fuerzas sociales, culturales y políticas que emanan de la Ciudad simbólica con el fin de modelar a la sociedad de la Reserva simbólica.

Las condiciones en que se abandona la Reserva simbólica son muy complejas, conflictivas y raramente claras para quien se desplaza. Con frecuencia, la motivación aparente en la supresión disimula razones más profundas que tienen implicaciones espirituales y políticas. Un indicador importante en el desarrollo del héroe de las narrativas de la supresión es de hecho, su comprensión del contexto profundo de la supresión misma. A primera vista, un individuo se desplaza o se suprime para unirse al ejército, como en Ceremony o en House Made of Dawn. Se suprimen para obtener una educación occidental, como en The failing of Cecelia Capture de Janet Campbell Hale. Se suprimen siguiendo la promesa de nuevas oportunidades y de una experiencia cosmopolita de la ciudad, como en Reservation Blues de Sherman Alexie. O se suprimen con el objeto de obtener poder económico para ayudar a sus familias, como en Halfbreed de María Campbell. Solo después de experimentar la vida en la Ciudad simbólica, obtienen la perspectiva crítica necesaria para comprender la influencia que ejercen las fuerzas del colonialismo y la modernidad sobre su modo de pensar y sobre la vida de la comunidad en la Reserva simbólica. En Halfbreed de Campbell, María desea una "mejor" vida en términos económicos, diferente de la que llevaba con su familia en las parcelas de tierra de los caminos, así como mantener unidos a sus hermanos y hermanas, a la vez que alejados de las autoridades provinciales que les vigilaban. Una vez que María se desplaza a la Ciudad simbólica, entiende la forma errada en que define esa "mejor" vida en los términos consumistas de la sociedad occidental. El articularse con las dinámicas de la Ciudad simbólica revela las verdades de la misma solo como teorías, sus juicios como sesgos, su compasión como mero paternalismo.

Las decisiones que un individuo toma al navegar ese nuevo mundo y al aprender su lenguaje determinan si llegará a ser un superviviente articulado con una voz propia y así, poder de decisión, o si se convertirá en un ser desarticulado, un superviviente sin autonomía alguna. Llamar "héroe" al protagonista de las narrativas de la supresión es de algún modo una contradicción, puesto que no se trata de un individuo semejante al de las nociones estereotípicas del héroe que continúa desempeñando un rol central en la cultura popular. Este personaje 
carece de las cualidades propias del heroísmo -la valentía marcial, el coraje, el sacrificio por el bien de su comunidad. Por lo contrario, para conseguir el conocimiento necesario de la Ciudad simbólica, el héroe de las narrativas de la supresión está obligado (en ocasiones de modo temporal, otras de manera permanente) a sacrificar aquellos valores extraídos del Centro simbólico por aquellos de la Ciudad. De este modo, Abel en House Made of Dawn, el narrador en Winter in the Blood de James Welch, y Manson Rollins en Smoke Dancing de Gansworth deben entenderse más como antihéroes que como héroes. Antes de volver a la Reserva simbólica, en plena búsqueda el héroe de las narrativas de la supresión se convierte en un egoísta, en un individualista, puesto que vive dentro del sistema de valores de la Ciudad simbólica. Ejemplo de esto es Ben Benally, quizá el superviviente articulado mejor descrito, quien reflexiona en la sección "The Night Chanter" de Una casa hecha de amanecer que hay razones para querer hacer parte de la Ciudad simbólica:

Ellos tienen bastantes palabras y uno sabe que significan algo, pero en principio uno no sabe qué significan, y tus propias palabras no son buenas porque no son lo mismo; son diferentes y son las únicas palabras que tienes. Todo es diferente y no sabes cómo acostumbrarte a todo ello. Entiendes cómo es todo aquello, cómo todo sigue adelante sin ti, y empiezas a preocuparte mucho. Te preguntas cómo puedes hacer parte de todo ello, ¿ ¿sabes? Y no sabes cómo, pero tienes que hacerlo porque no hay nada más. Y quieres hacerlo porque sientes lo bueno que puede ser. Es mejor que cualquier cosa que te haya pasado antes; se trata de dinero y prendas de vestir, y de tener planes e ir velozmente a algún sitio. (Momaday, House 139)

Para Ben, regresar a la Reserva simbólica no es una opción. Puede llegar a considerarlo bajo los efectos del alcohol, "pero al siguiente día sabes que no sirve de nada; sabes que si volvieras a casa no habría nada para ti allí, sólo una tierra vacía y un puñado de gente sin a donde ir y que se muere en la espera" (Momaday, House 140). Mientras Ben quiere encontrar una comunidad en la Reserva simbólica, en la ciudad oscila entre la soledad y la alienación: las dos alternativas propuestas por Stepto para el superviviente articulado.

El superviviente articulado se ha educado en el lenguaje, los códigos y las leyes de la Ciudad simbólica. Puede llegar a adaptarse a la modernidad y a la libertad relativa que la ciudad ofrece por vías de un proceso de asimilación a los preceptos imperantes en ese tipo de sociedad. En un caso extremo, el individuo puede hacerse pasar como no indígena de manera consciente o inconsciente. Pero con más frecuencia, el individuo vive una existencia escindida; construyendo de 
manera consciente una apariencia no indígena en términos culturales, a la vez que un carácter cultural indígena que se expresa en la vida privada. El superviviente articulado se precia de saber cómo se vive en la Ciudad simbólica, pero se encuentra angustiado por el prospecto de haber aprendido las reglas tan bien que se ha convertido en un sujeto dominado por el poder de la ciudad. Constantemente debe enfrentarse al hecho de haber sacrificado su sentido de indigenidad al entrar en complicidad con los valores de la ciudad. Al saber esto, el héroe busca disimular las verdaderas razones por las cuales otros son incapaces de sobrevivir en la ciudad. En House Made of Dawn, Ben explica la incapacidad de Abel para adaptarse a Los Angeles asegurando que "no tuvo suerte" (Momaday, House 139).

Si el superviviente articulado domina el lenguaje de la Ciudad simbólica, el superviviente desarticulado es un individuo que pierde la voz y que en consecuencia, carece de toda potestad sobre sí en la ciudad. En las literaturas indígenas, el superviviente desarticulado puede haber aprendido el lenguaje de la Ciudad simbólica pero debido a variadas razones, se niega a emplearlo. Con frecuencia este individuo aprenderá las idiosincrasias de ese sistema solo para sobrevivir de manera muy precaria en esta zona simbólica. El tráfico cultural y las demandas de la ciudad que exigen grandes sacrificios -abandono de la familia, de la comunidad, de la autoestima, del pensamiento político, de las creencias, de la dignidad-para alcanzar el "éxito", en su momento, se vuelven insoportables; la disonancia cognitiva del individuo se refleja en la pérdida de una voz, de una comunicación coherente. Para personajes como Helen Jean en Ceremony, la vergüenza de todo aquello que se ha perdido puede dejar encadenado a perpetuidad al personaje a la Ciudad simbólica. Ese personaje mudo puede sobrevivir en la marginalidad al interior de la ciudad, en las calles y en los muelles, como Jackson Jackson hace en el cuento de Sherman Alexie "What You Pawn I Will Redeem", quien se encuentra loco y narcotizado. Separado del sentido nutricio de la comunidad, el superviviente inarticulado está condenado a languidecer en la ciudad o, como ocurre con frecuencia en las literaturas indígenas, a iniciar el proceso de retorno hacia la vida comunitaria de la Reserva simbólica y su fuente crítica moralizante, el Centro simbólico.

Las narrativas del retorno se estructuran a partir de una búsqueda individual para reintegrar al individuo fragmentado y de una búsqueda de la comunidad que quiere fortalecer y reunificar a un pueblo a través de una reafirmación de la capacidad de sus tradiciones para resistir a la Ciudad simbólica. Una vez que el héroe de las narrativas de la supresión se encuentra inmerso en la Ciudad simbólica, se le presenta un dilema que debe resolver: ¿permanecerá en la ciudad o retornará a la Reserva simbólica? Es posible que el superviviente articulado sea asimilado y absorbido en el sistema de la Ciudad simbólica. No obstante, un movimiento de 
alejamiento permanente de cualquier comunidad de Reserva simbólica es inusual en las literaturas indígenas. Tal es el caso con la supresión, las razones prácticas para regresar a la Reserva simbólica disimulan preocupaciones más profundas. Lena en Miko Kings: An Indian Baseball Story, de Le Anne Howe, se ve convocada de vuelta a la comunidad por los ancestros, lo cual en sí mismo se convierte en una afrenta a los sentimientos de un superviviente articulado que rehúye las tradiciones. En Bone Game de Louis Owens el compromiso involuntario de Cole McCurtain con el mundo de los espíritus se convierte en la narrativa a través de la cual el héroe decide permanecer como un superviviente articulado o convertirse en un individuo articulado a su clan. En cualquier caso, superviviente articulado o desarticulado, el regreso a la Reserva simbólica inicia una transformación del ser por medio de la que el personaje debe compartir, así como emplear, su conocimiento de la Ciudad simbólica, a la vez que reaprende las costumbres del Centro simbólico.

Un retorno exitoso exige del protagonista un juicio apropiado acerca del conocimiento que llevará a la Reserva simbólica. Su experiencia en la Ciudad simbólica le ayudará a elegir entre aquello que resultará beneficioso y aquello que puede ser potencialmente dañino para la comunidad. La reintegración satisfactoria de un personaje a la Reserva simbólica depende de su capacidad para deshacerse de los valores individuales de la Ciudad simbólica, así como de los prejuicios con respecto a la Reserva y el Centro simbólicos. A menudo, las suposiciones que hace un individuo acerca del poder cultural y la eficacia espiritual del Centro simbólico se encuentran alteradas por el conocimiento obtenido en la Ciudad simbólica. Personajes como Tayo en Ceremony o Lisa en Monkey Beach desconfían de la autenticidad de sus experiencias con el mundo de los espíritus. O, como el narrador en The Way to Rainy Mountain de Momaday, que debe aprender a equilibrar las representaciones orales y escritas de su comunidad al suprimir la importancia del texto escrito - un aspecto preponderante de la ciudad- y al enaltecer la tradición oral. Los vínculos abstractos y teóricos con la realidad que pone de relieve la Ciudad simbólica deben equilibrarse con el conocimiento obtenido por medio de la experiencia. Ultimadamente no obstante, no existe una fórmula para equilibrar las influencias de la Ciudad y del Centro simbólicos; cada retorno requiere diferentes aspectos de cada individuo y su comunidad. En The Death of Fim Loney, James Welch describe a un superviviente desarticulado que no puede reintegrarse a la comunidad de la Reserva simbólica de su niñez. En Los Hiawatha de David Treuer se desarrolla un ciclo de supresión y retorno que también fracasa. Las muchas maneras en que los personajes triunfan o fracasan a este respecto es lo que en parte hace que las narrativas del retorno sean tan atractivas. 
Los textos indígenas retratan al individuo articulado a su clan como un personaje que se encuentra tan bien fundamentado en el lenguaje de su Centro simbólico que puede involucrarse y aprender acerca del mundo occidental de la Ciudad simbólica sin temor a perder su voz. Como Betonie en Ceremony, el individuo articulado a su clan se convierte en un mediador cultural que entiende ambos marcos de pensamiento y la manera en que se dinamizan con la Reserva simbólica. Esto se ve reflejado en la manera en que Betoine asegura que existe una necesidad de cambiar los rituales tribales para entrar en consonancia con un mundo mutable, "he hecho cambios en los rituales. El pueblo desconfió mucho de esto, pero sólo este crecimiento mantiene fuertes las ceremonias" (Silko 116). William Listener en The Remnants of the First Earth de Young Bear, propone un modelo del individuo articulado al clan a través de los lenguajes hablados de la Ciudad, de la Reserva y del Centro simbólicos:

Cuando no estaba orando, estaba cantando y tocando el tambor. Cuando no estaba realizando una complicada ceremonia de contacto con la tierra, leía anotaciones en el cónclave tribal del Niño Águila Negra o instalando tuberías en el pueblo. William fue la primera persona en demostrar que uno podía educarse en ambos mundos al tener un control total de sus diversos lenguajes. La posesión de una perspectiva perspicaz y analítica, pero tradicional fue también de gran ayuda. (Young Bear 116)

Tal como lo muestran los modelos de Listener y Betoine, en los mundos narrativos de las literaturas indígenas los cambios sociales, culturales y políticos que el colonialismo y la modernidad han causado en las comunidades indígenas deben tomarse en cuenta si se quiere que el Centro simbólico sobreviva. Para poder considerar una continuidad de la modernidad y el colonialismo, el conocimiento de la Ciudad simbólica debe llevarse a las comunidades indígenas que se resisten en la Reserva simbólica. Los procesos críticos y los valores del Centro simbólico dependen así, del conocimiento y las experiencias de los protagonistas de las narrativas del retorno. Sin este conocimiento, la comunidad de la Reserva simbólica se arriesga a perder su soberanía cultural y política. Sus tradiciones tribales pueden perder eficacia en contrarrestar el colonialismo y la modernidad. A su regreso, el protagonista afirma su necesidad de estar en la comunidad de la Reserva simbólica así como el poder del Centro simbólico.

\section{La experiencia indígena y las geografías simbólicas}

El marco geográfico simbólico que propongo en este ensayo le ofrece a los estudios críticos indigenistas un marco que debería completarse, cuestionarse y 
elaborarse para reflejar la riqueza y la diversidad de los discursos indígenas. $\mathrm{Si}$ se reconocen las narrativas de remoción y retorno, puede deberse en parte, a que nos resultan familiares en la vida diaria. Los patrones literarios se modelan con frecuencia desde la vida cotidiana. Muchos de nosotros, nuestras familias y amigos, nos encontramos comprometidos a diario en una negociación con el Centro simbólico, la Reserva simbólica y la Ciudad simbólica. Puede que nos encontremos gravitando entre estas existencias, fortaleciéndonos unos a otros en un constante reconstruir de comunidad, robusteciendo nuestros universos con conocimiento, y encontrando nuestro centro con la tradición. O podríamos encontrarnos en otro punto del ciclo de remoción y retorno, sopesando nuestras elecciones, evaluando nuestras voces. En cualquier caso, las elecciones que los personajes indígenas hacen ofrecen algún tipo de guía.

Como en la articulación de personas del mismo linaje, los académicos de las literaturas indígenas han estado acudiendo a múltiples recursos críticos, tanto indígenas como no indígenas. Meditamos los diferentes lenguajes de nuestra experiencia para crear teorías y modelos críticos que se espera, proveerán tanto a nuestros lectores de la Reserva simbólica como a aquellos de la Ciudad simbólica, de herramientas críticas que necesitan para escucharse y comunicarse entre sí a través del velo. La teoría de Trickster, el feminismo indígena, los nacionalismos literarios y los acercamientos críticos centrados en lo tribal sirven como ejemplos de este esfuerzo. Los Centros simbólicos indígenas crecen y mutan, allí radica su poder y en la medida en que lo hacen, deben tomar conciencia total del mundo que las comunidades indígenas experimentan, aprovechando de esta manera la experiencia tribal en su totalidad.

\section{Obras citadas}

Alexie, Sherman. Reservation Blues. New York: Warner Books, 1996.

-. Ten Little Indians. Nueva York: Grove, 2003.

Bevis, William. "Native American Novels: Homing". Recovering the Word:

Essays on Native American Literature. Brian Swann y Arnold Krupat.

Ed. Berkeley: University of California Press, 1987, 580-620.

Campbell, Maria. Halfbreed. Lincoln: University of Nebraska Press, 1982/1973.

DuBois, W.E.B. The Souls of Black Folk. Nueva York: Vintage, 1990/1903.

Gansworth, Eric. Smoke Dancing. Lansing: Michigan State University Press, 2004.

Hale, Janet Campbell. The failing of Cecelia Capture. Albuquerque:

University of New Mexico Press, 1993/1985.

Highway, Tomson. Kiss of the Fur Queen. Norman: University of Oklahoma Press, 2000. Howe, LeAnne. Miko Kings: An Indian Baseball Story. San Francisco: Aunt Lute, 2007. 
-. Shell Shaker. San Francisco: Aunt Lute, 2001.

King, Thomas. Truth \& Bright Water. Nueva York: Grove, 1999.

LaDuke, Winona. Last Standing Woman. Stillwater, MN: Voyageur, 1997.

McNickle, D'Arcy. The Surrounded. Albuquerque:

University of New Mexico Press, 1997/1936.

Momaday, N. Scott. The Way to Rainy Mountain. 1969.

Albuquerque: University of New Mexico, 1995.

-. House Made of Dawn. Nueva York: Perennial Classics, 1999/1968.

Neihardt,John G. Black Elk Speak: Being the Life of a Holy Man of the

Oglala Sioux. Lincoln: University of Nebraska Press, 1989/1932.

Owens, Louis. Bone Game. Norman: University of Oklahoma Press, 1994.

-. Other Destinies: Understanding the American Indian Novel.

Norman: University of Oklahoma Press, 1992.

Parker, Robert Dale. The Invention of Native American

Literature. Ithaca: Cornell University Press, 2003.

Power, Susan. The Grass Dancer. Nueva York: Penguin, 1995.

Robinson, Eden. Monkey Beach. Nueva York: Houghton Mifflin, 2000.

Robinson, Harry. "An Okanagan Indian Becomes a Captive Circus Showpiece in

England". All My Relations: An Anthology of Contemporary Canadian Native

Fiction. Thomas King. Ed. Toronto: McClelland \& Stewart, 1990. 1-26

Silko, Leslie Marmon. Ceremony. Nueva York: Penguin, 2007/1977.

Stepto, Robert B. From Behind the Veil: A Study of Afro-American

Narrative. Urbana: University of Illinois Press, 1979.

Teuton, Sean Kicummah. Red Land, Red Power: Grounding Knowledge in

the American Indian Novel. Durham: Duke University Press, 2008.

Treuer, David. The Hiawatha. Nueva York: Picador, 1999.

Van Camp, Richard. The Lesser Blessed. Vancouver: Douglas \& McIntyre, 1996.

Vizenor, Gerald. Dead Voices: Natural Agonies in the New World.

Norman: University of Oklahoma Press, 1992.

Welch, James. Winter in the Blood. Nueva York: Penguin, 1986/1974.

- . The Death of Fim Loney. Nueva York: Penguin, 1979.

-. The Heartsong of Charging Elk. Nueva York: Doubleday, 2000.

Werner, Craig Hansen. Playing the Changes: From Afro-Modernism to

the fazz Impulse. Urbana: University of Illinois Press, 1994.

Wilson, Michael. "Speaking of Home: The Idea of the Center in Some

Contemporary American Indian Writing". WicazoSa Review (1997): 129-147

Young Bear, Ray A. Remnants of the First Earth. Nueva York: Grove, 1996. 